\title{
Molecular analysis of Physarum haemagglutinin I: lack of a signal sequence, sulphur amino acids and post-translational modifications
}

\author{
Masashi Morita \\ Tel: +81764342281 ext. 2636. Fax: +81764344656. \\ e-mail:masa@ms.toyama-mpu.ac.jp
}

Faculty of Pharmaceutical Sciences, Toyama Medical and Pharmaceutical University, Sugitani, Toyama, 930-01, Japan

\begin{abstract}
The cDNAs encoding haemagglutinin I from plasmodia of Physarum polycephalum have been cloned using PCR protocols. The composite haemagglutinin I CDNA sequence, derived from several overlapping clones from PCR fragments, spans $408 \mathrm{nt}$ and the $\mathbf{3 1 5}$ bp ORF encodes a polypeptide of 104 aa without a typical signal sequence. The putative molecular mass deduced from the amino acid sequence (10760.76 Da) corresponds exactly to that determined by electrospray ionization MS (10759.86 $\pm 0.15 \mathrm{Da})$, suggesting that haemagglutinin I is not subject to post-translational modification. Haemagglutinin I lacks sulphur amino acids and has a $\beta$-sheet as the major secondary structure. Expression of the coding sequence in Escherichia coli yielded a product that exhibits the same sugar-binding specificity as natural haemagglutinin $I$. The deduced amino acid sequence shows little similarity to that of any known lectins and thus apparently represents a novel type of lectin.
\end{abstract}

Keywords: haemagglutinin, lectin, Physarum polycephalum, cDNA cloning, posttranslational modification

\section{INTRODUCTION}

Lectins are a class of proteins that agglutinate erythrocytes (so-called haemagglutinins) and the agglutination is inhibited by adding monosaccharide, oligosaccharide and/or glycoprotein. Lectins bind stereospecifically and reversibly to a carbohydrate and act as mediators of various physiological cellular events. To date, many amino acid sequences of lectins in higher plants, animals, fungi and bacteria have been revealed. Despite numerous studies and their wide distribution in nature, the physiological functions of these proteins have not yet been clearly established.

Several fungal lectins have been identified and characterized. Discoidins I and II, which are lectins produced by the cellular slime mould Dictyostelium discoideum,

Abbreviations: ESI-MS, electrospray ionization MS; RACE, rapid amplification of CDNA ends; RP-HPLC, reverse-phase HPLC; TFA, trifluoroacetic acid.

The GenBank accession number for the sequence reported in this paper is U86761. have been reported to be involved in cell-substratum attachment (Barondes \& Springer, 1987) and in spore coat formation (Cooper et al., 1983), respectively. In contrast, a lectin in the nematophagous fungus Arthrobotrys oligospora has been reported to be involved in the capture of nematodes (Rosen et al., 1996a, b). Lectins from plant-pathogenic fungi such as Sclerotium rolfsii and Rhizoctonia solani function in the recognition and attachment of these fungi by the mycoparasite Trichoderma (Inbar \& Chet, 1994). A previous study (Morita \& Nishi, 1992) has shown that the acellular slime mould Physarum polycephalum produces two types of lectins, termed haemagglutinins I and II, which differ in terms of their molecular masses and carbohydrate-binding properties. These haemagglutinating activities were not inhibited by various monosaccharides but were strongly inhibited by thyroglobulin. We have also reported that an acidic polysaccharide from the bacterial cell wall might be a relevant ligand for haemagglutinin (Morita et al., 1995) and have hypothesized that Physarum haemagglutinins might play a physiological role in the interaction with bacteria in nature. However, the apparent role of haemagglutinins is not yet clear. 
The slime mould is a suitable organism for the study of cell growth and development at the biochemical and molecular levels. It has been reported that the expression of numerous genes is regulated during the Physarum life cycle (Schreckenbach \& Werenskiold, 1986). The expression of haemagglutinins is thought to be developmentally regulated because haemagglutinating activities have been found in the plasmodial stage (diploid) but not in the myxoamoebae stage (haploid). Determination of the amino acid sequence of haemagglutinin I is needed to obtain more information on the role of the protein and to understand its sugar-binding specificity. Here we report the determination of the complete nucleotide sequence of haemagglutinin I CDNA and the expression of recombinant active haemagglutinin I in Escherichia coli.

\section{METHODS}

Cultivation of microplasmodia. Microplasmodia of $P$. polycephalum were grown in a semi-defined medium containing glucose, yeast extract, tryptone and haemin (Daniel \& Baldwin, 1964). The axenic culture was maintained in $100 \mathrm{ml}$ liquid medium and shaken at $26^{\circ} \mathrm{C}$ in the dark.

Purification of haemagglutinin I. Purification of haemagglutinin I from microplasmodia was performed as described previously (Morita \& Nishi, 1992). Haemagglutinating activity and haemagglutination inhibitory activity were measured with trypsin-treated, formalin-fixed rabbit erythrocytes as described previously (Morita et al., 1995).

Reverse-phase (RP) HPLC. Purified haemagglutinin I $(60 \mu \mathrm{g})$ was treated with $8 \mathrm{M}$ urea in $0.1 \mathrm{M}$ ammonium bicarbonate, $\mathrm{pH} 8 \cdot 0$, containing $2 \mathrm{mM} \beta$-mercaptoethanol for $45 \mathrm{~min}$ at $60^{\circ} \mathrm{C}$. When the concentration of urea reached $2 \mathrm{M}$, tryptic or chymotryptic cleavage was carried out with trypsin (Seikagaku Kogyo) or bovine pancreas chymotrypsin VII (Sigma), respectively, at an enzyme/substrate ratio of $1: 50(\mathrm{w} / \mathrm{w})$ at $37^{\circ} \mathrm{C}$ for $3 \mathrm{~h}$. After lyophilization, the resultant peptides were dissolved in $30 \mu \mathrm{l} 0.1 \%(\mathrm{v} / \mathrm{v})$ trifluoroacetic acid (TFA) and applied to a Cosmosil 5C18-MS (C18) reverse-phase column $(4 \cdot 6 \times 150 \mathrm{~mm}$, Nacalai Tesque) equilibrated with $0 \cdot 1 \%$ TFA for HPLC. Peptide fragments were separated using the following gradient of acetonitrile in $0.1 \%$ TFA: $10 \%$ at $0 \mathrm{~min}$, followed by a linear gradient to $55 \%$ at $40 \mathrm{~min}$ and finally a linear gradient to $80 \%$ at $50 \mathrm{~min}$. Peptides were detected at $A_{220}$. Peptide fragments were collected manually over several runs and the pure peptides were sequenced using a Shimadzu PPSQ-10 protein sequencer.

In another experiment, purified haemagglutinin I ( $300 \mu \mathrm{g})$ was denatured in $6 \mathrm{M}$ guanidine hydrochloride and subjected to RP-HPLC, as above. Haemagglutinin I was eluted with a linear gradient $(0-80 \%)$ of acetonitrile in $0.1 \%$ TFA at a flow rate of $0.8 \mathrm{ml} \mathrm{min}^{-1}$ for $50 \mathrm{~min}$. Elution was monitored at $A_{280}$.

Tricine-SDSPAGE and blotting. Purified haemagglutinin I was subjected to Tricine-SDS-PAGE according to the procedure of Schagger \& van Jagow (1987) as described previously (Morita \& Nishi, 1992). For peptide sequencing, haemagglutinin I was transferred to a PVDF membrane $(0 \cdot 2 \mu \mathrm{m}$, Bio-Rad) using a semi-dry electroblotting system (Bio-Rad) and applied directly to an automated protein sequencer according to the procedure of Matsudaira (1987).
Electrospray ionization MS (ESI-MS). The molecular masses of protein or peptide were determined by ESI-MS using an AP-III triple quadrupole mass spectrometer (Perkin-Elmer Sciex Instruments). Calibration of the mass range was achieved by injecting a mixture of PEGs. RP-HPLC-purified proteins or peptides were dissolved in $50 \%$ acetonitrile containing $0 \cdot 1 \%$

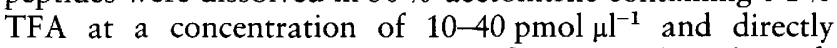
introduced into the ion source at a flow rate of $20 \mu \mathrm{l} \mathrm{min}-1$. The molecular mass spectrum was reconstructed from multiply charged ions in the $m / z$ spectrum.

Purification of poly(A) RNA. Unless stated otherwise, all reagents used were treated with diethylpyrocarbonate. Total RNA was extracted from vegetatively growing microplasmodia of $P$. polycephalum according to the procedure of Nishimura et al. (1991). Isolation of poly(A) RNA from the total RNA was carried out using Oligotex-dT30 (Takara) according to the manufacturer's protocol.

cDNA cloning and sequencing. CDNA cloning was done using PCR-based protocols with primers given in Table 1 . Restriction digests, DNA ligation and plasmid DNA preparation from $E$. coli were done using standard protocols described in Sambrook et al. (1989). First-strand cDNA was prepared by reverse transcription of poly(A) RNA using SuperScript TM II RT (Gibco-BRL) according to the manufacturer's protocol with oligo(dT)-containing Adapter Primer (Gibco-BRL). PCR was performed with the first-strand $\mathrm{cDNA}$ as the template using two sets of oligonucleotide mixture designed on the basis of the N-terminal 65 aa sequence. Amplification with primers 1 and 2 produced polynucleotides of approximately $200 \mathrm{bp}$. This product was then used as template for nested PCR performed with primers 3 and 4 . Nested PCR yielded an amplification product of $180 \mathrm{bp}$. To obtain the $3^{\prime}$ or $5^{\prime}$ ends, $3^{\prime} \mathrm{RACE}$ or $5^{\prime} \mathrm{RACE}$ (rapid amplification of cDNA ends) was performed according to the procedure of Frohman et al. (1988). Using the 3'RACE System (Gibco-BRL), oligo(dT)primed cDNA was amplified with Abridged Universal Amplification Primer (supplemented by the RACE System) and primer 5. The 5'RACE System (Gibco-BRL) was utilized to synthesize first-strand cDNA for the $5^{\prime}$ end with primer 6 , followed by ligation of the Abridged Anchor Primer. PCR was performed with primer 7 and Abridged Universal Anchor Primer to yield an amplification product. Nested PCR was then performed with the amplification product as the template using primer 8 and Abridged Universal Anchor Primer. All PCR amplifications were performed with Taq DNA polymerase (Gibco-BRL) in the buffer composition and other conditions suggested by the manufacturer. PCR was performed on a Perkin-Elmer Cetus DNA thermal cycler (model 480) with 30 cycles of $94^{\circ} \mathrm{C}$ for $45 \mathrm{~s}$ (denaturation), $55^{\circ} \mathrm{C}$ for $25 \mathrm{~s}$ (annealing) and $72^{\circ} \mathrm{C}$ for $1 \mathrm{~min}$ (extension). After the final cycle, DNA was further extended at $72^{\circ} \mathrm{C}$ for $7 \mathrm{~min}$.

Each amplified PCR fragment was isolated by electrophoresis using low-melting-point agarose and was directly cloned into the pT7Blue(R)T-vector (TA-cloning system, Novagen). The recombinant plasmid was transformed into $E$. coli XL-1 Blue

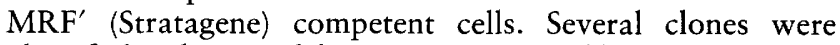
identified and some of them were sequenced by a conventional dideoxy chain-termination method (Sanger et al., 1977) using Sequencing High -Cycle- (Toyobo) and Sequenase Images (Amersham) according to the manufacturer's protocol. Several clones were sequenced in both directions.

Computer analysis. The GENETYX-sv/DB (Software Development) program was used for analysis of the primary and secondary structure of the protein. The SWISS-PROT and 
Table 1. Oligonucleotides used for the cloning of haemagglutinin I CDNA

\begin{tabular}{|clcc|}
\hline Primer & \multicolumn{1}{c|}{ Sequence $\left(\mathbf{5}^{\prime} \mathbf{3}^{\prime}\right)^{*}$} & Direction† & $\begin{array}{c}\text { Target } \\
\text { nucleotides } \neq\end{array}$ \\
\hline 1 & GTNTGGWSNGTNCARATHGTNGAY & Sense & $34-57$ \\
2 & RTACCANSWYTGRTGNACNGTYTT & Antisense & $195-172$ \\
3 & GTNCARATHGTNGAYAAYGCNGGN & Sense & $43-66$ \\
4 & YTGRTGNACNGTYTTNGGNCCDATYTC & Antisense & $186-160$ \\
5 & AATTCTTCCACTGTCCCTCG & Sense & $100-119$ \\
6 & GAGGGTCAAATGCTGGG & Antisense & $330-314$ \\
7 & ATACTCACATTCCCACCTGCTG & Antisense & $293-272$ \\
8 & TGCTCTGTCATCTCCATTGTGC & Antisense & $225-204$ \\
9 & GAAGCATATGGTCTGGTCGGTGCAAATA & Sense & $24-51$ \\
10 & TTGTGGATCCTTATGCAAGTTTCTTGAG & Antisense & $355-328$ \\
\hline
\end{tabular}

*D denotes A or G or T; M, A or C; R, A or G; S, C or G; W, A or T; Y, C or T. Sequences of primers $1,2,3$ and 4 were designed on the basis of 65 aa $\mathrm{N}$-terminal sequences. Restriction enzyme recognition sites incorporated into primer sequences are indicated by underlining ( $N d e \mathrm{I}$ in primer 9; Bam $\mathrm{HI}$ in primer 10).

† With respect to the sequence shown in Fig. 1.

$\neq$ Corresponding cDNA sequence numbered as shown in Fig. 1.

NBRF protein sequence databases and GenBank were used for sequence homology searching. The prediction of the hydropathy profile was performed according to the method of Kyte \& Doolittle (1982) with a window size of eight residues. The prediction of secondary structure was made using the program of Chou \& Fasman (1978).

Northern blot analysis. Northern blot analysis was performed according to the standard method (Sambrook et al., 1989) using total RNA prepared from microplasmodia. About $15 \mu \mathrm{g}$ total RNA was separated by electrophoresis on a $1 \%(\mathrm{w} / \mathrm{v})$ agarose $/ 2 \cdot 2 \mathrm{M}$ formaldehyde gel and transferred onto a nylon membrane (Hybond- $\mathrm{N}^{+}$, Amersham) by capillary action. The filter was hybridized with fluorescein-labelled probe, prepared using Gene Images (Amersham) by specific primer extension labelling. A $0 \cdot 2 \mathrm{kbp}$ cDNA fragment amplified with primers 5 and 7 was labelled with fluorescein-dUTP using primer 8 and used as probe. After hybridization the blot was treated with an alkaline-phosphatase-labelled anti-fluorescein antibody followed by treatment with detection reagents and the filter was exposed to XAR-5 film (Kodak) for $2 \mathrm{~h}$. An RNA ladder $(0 \cdot 16-1 \cdot 77 \mathrm{~kb})$ was used as a marker.

Expression of haemagglutinin $\mathbf{I}$ in $\boldsymbol{E}$. coli. Expression of recombinant protein was done using the $\mathrm{pET}-3$ a vector in the BL21 host (pET Expression System 3, Novagen). DNA fragments containing an $\mathrm{NdeI}$ site covering the start codon and a BamHI site downstream of the desired stop codon were generated by PCR using primers 9 and 10, as shown in Table 1. After digestion with $N d e \mathrm{I}$ and $B a m \mathrm{HI}$, the PCR-generated DNA fragment was inserted between the same sites of pET-3a. E. coli NovaBlue (Novagen) was used as a host for the first cloning. The cloned plasmid was then introduced into $E$. coli BL21(DE3)plysS cells grown on LB plates containing $50 \mu \mathrm{g}$ ampicillin $\mathrm{ml}^{-1}$ and $15 \mu \mathrm{g}$ chloramphenicol $\mathrm{ml}^{-1}$. The BL21(DE3)plysS cells containing the recombinant plasmid were grown to mid-exponential phase at $37^{\circ} \mathrm{C}$ in LB medium, induced by adding IPTG to a concentration of $0.4 \mathrm{mM}$ and incubated at $26^{\circ} \mathrm{C}$. After an additional $3 \mathrm{~h}$ culture period, $E$. coli cells were collected by centrifugation and resuspended in $50 \mathrm{mM}$ Tris $/ \mathrm{HCl}, \mathrm{pH} 8.0$, containing $0.5 \mathrm{mM}$ EDTA, $0.4 \mathrm{M}$
$\mathrm{NaCl}, 5 \mathrm{mM} \mathrm{MgCl}_{2}, 5 \%$ (w/v) glycerol, $0.5 \mathrm{mM}$ PMSF, $1 \mathrm{mM}$ DTT and $1 \mathrm{mg}$ lysozyme $\mathrm{ml}^{-1}$. Then the cell lysate was prepared by repeated freezing and thawing, and sonicated for $20 \mathrm{~s} 10$ times with an Ultrasonic Disrupter (model UR-200P, Tomy Seiko) after the addition of EDTA and Nonidet P40 to final concentrations of $1 \mathrm{mM}$ and $0.5 \%(\mathrm{w} / \mathrm{v})$, respectively. The cell lysate was centrifuged at $15000 \mathrm{~g}$ for $30 \mathrm{~min}$ to remove insoluble materials. After desalting by ultrafiltration (PM 10, Amicon) the supernatant was applied to a column of DEAE-Sephacel $(3 \times 15 \mathrm{~cm}$, Pharmacia) equilibrated with $10 \mathrm{mM}$ Tris/ $\mathrm{HCl}, \mathrm{pH} 7 \cdot 5$. The column was washed with the same buffer to remove unadsorbed materials and subsequently the adsorbed proteins were eluted with the same buffer containing $1 \mathrm{M} \mathrm{NaCl}$. Those exhibiting haemagglutinating activity were pooled and the active fraction was further purified using a thyroglobulin-immobilized Toyopearl HW55 column $(3 \times 15 \mathrm{~cm}$, Tosoh) as described previously (Morita $\&$ Nishi, 1992). In some experiments, the pellet of intact bacteria was directly solubilized in sample buffer and analysed by Tricine-SDS-PAGE.

\section{RESULTS}

\section{Partial amino acid sequence}

Our previous study (Morita \& Nishi, 1992) reported that plasmodia of $P$. polycephalum produce two types of lectins, haemagglutinins I and II, which migrate to about 6 and $11 \mathrm{kDa}$ upon Tricine-SDS-PAGE, respectively. In this study, direct Edman analysis of the band migrating to $6 \mathrm{kDa}$ revealed the sequence of the first $23 \mathrm{~N}$-terminal residues (Table 2). To obtain additional sequence, haemagglutinin I was subjected to tryptic or chymotryptic digestion and the resultant peptides were analysed by Edman degradation and ESI-MS after purification with RP-HPLC (Table 2). ESI-MS can measure molecular masses of polypeptide chains to an accuracy of about $0.01 \%$. Therefore, we applied this method to confirm the amino acid sequence determined by Edman 
Table 2. Amino acid sequences and masses of intact haemagglutinin I, and fragments generated from purified haemagglutinin I by trypsin and chymotrypsin treatment

Haemagglutinin I and its tryptic fragments purified by RP-HPLC on a $\mathrm{C}_{18}$ column were subjected to MS to determine their molecular mass. ND, not determined.

\begin{tabular}{|lc|}
\hline Sequence & $\begin{array}{c}\text { Molecular mass } \\
\text { from ESI-MS (Da) }\end{array}$ \\
\hline Intact haemagglutinin I* & \\
VWSVQIVDNAGLGANLALYPSGN------ & $10759 \cdot 86 \pm 0 \cdot 15$ \\
Trypsin fragments $\dagger$ & \\
Major peak & \\
T3 TVHQSWY & $919 \cdot 44 \pm 0 \cdot 08$ \\
T4 TVHQSWYITVHNGDDR & $1927 \cdot 68 \pm 0 \cdot 14$ \\
T6 YVTVTGYAPITFSEIGPK & $1943 \cdot 14 \pm 0 \cdot 35$ \\
T8 VWSVQIVDNAGLGANLALYPSGNSSTVPR & $2986 \cdot 73 \pm 0 \cdot 36$ \\
Minor peak & \\
T1 KLA & ND \\
T2 ITVHNGDDR & ND \\
T5 TAGGNVSISTGFGDAQHLTLK & $1331 \cdot 49 \pm 0 \cdot 70$ \\
T7 YVTVT------ & \\
Chymotrypsin fragments $\dagger$ & \\
Major peak & \\
C1 Negative & \\
C2 Negative & \\
C5 VW & \\
C6 SEIGPKTVHQSW------ & \\
C7 SEIGPKTVHQSWY----- & \\
C9 APITF----- & \\
C10 AL----- & \\
C11 ALYPSGNSSTVPRYV----- & \\
C13 ALYPS---- & \\
C14 SVQIVDNA---- & \\
Minor peak & \\
C3 ALYPSGN---- & \\
C4 ITVHNGDDRAF---- & \\
C8 IVDNAGLGA---- & \\
C12 Negative & \\
\hline
\end{tabular}

*Haemagglutinin I blotted on PVDF membrane was directly analysed by an automated protein sequencer.

†Trypsin (T) or chymotrypsin (C) fragments were numbered in accordance with their elution time on RP-HPLC. Dashes indicate that the peptide was not analysed further.

degradation. Digestion of haemagglutinin I with trypsin yielded eight fragments from which most of the $\mathrm{N}$ terminal sequence could be established. The molecular masses of the T3, T4, T5, T6 and T8 fragments, as determined by ESI-MS, were in good agreement with those calculated from the amino acid sequences $(919.97$, $1928 \cdot 02,2074 \cdot 25,1943 \cdot 17$ and $2986 \cdot 29 \mathrm{Da}$, respectively). Peptide T8 showed a sequence corresponding to a region in the N-terminal part of haemagglutinin I. Most of the peptide fragments were preceded by arginine or lysine, whereas T3 was preceded by tyrosine. This unexpected cleavage may be due to an abnormal action of the enzyme. Tryptic fragments T8, T6 and T4 overlapped with chymotryptic fragments $\mathrm{C} 11, \mathrm{C} 7$ and $\mathrm{C} 4$, which led to an unambiguous identification of residues 1-65 (Fig.
1). ESI-MS analysis of intact haemagglutinin I (Table 2) suggested that there should be about another $30 \mathrm{aa}$ residues in the $\mathrm{C}$-terminal region.

\section{Cloning of CDNA encoding haemagglutinin I}

Based on the N-terminal 65 aa sequence of haemagglutinin I, two sets of degenerate oligonucleotide primers (1 and 2, 3 and 4) were designed and used to amplify internal cDNA fragments. Several clones were identified and some of them were sequenced on both strands. Using primers designed from the cloned internal cDNA fragments, $5^{\prime}$ and $3^{\prime}$ end clones were isolated by RACE methods. The sequence of these clones together with internal cDNA fragments showed significant overlap- 


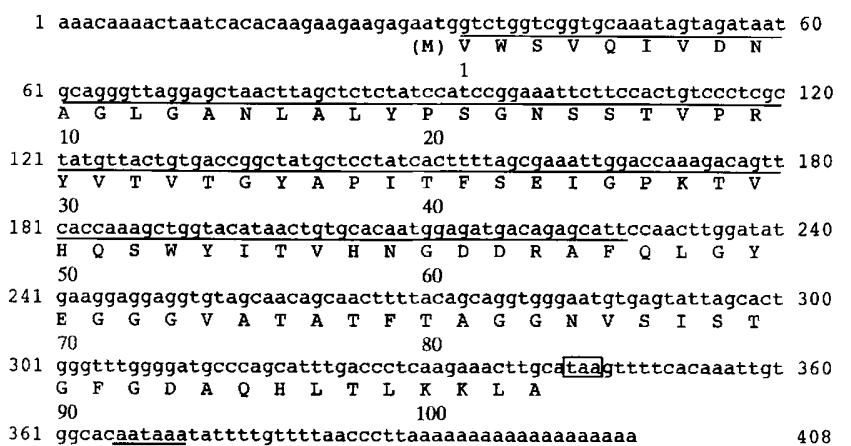

Fig. 1. The CDNA nucleotide sequence of Physarum haemagglutinin $\mid$ and its deduced amino acid sequence. Nucleotide numbers are shown on both sides of each line. The amino acid sequence is shown below the nucleotide sequence. The number of amino acid residues starting from $\mathrm{Val}$ is given below the amino acid sequence. The $\mathrm{N}$-terminal 65 aa sequence determined by Edman degradation is overlined. The box and double underlining denote the termination codon and the polyadenylation signal, respectively.

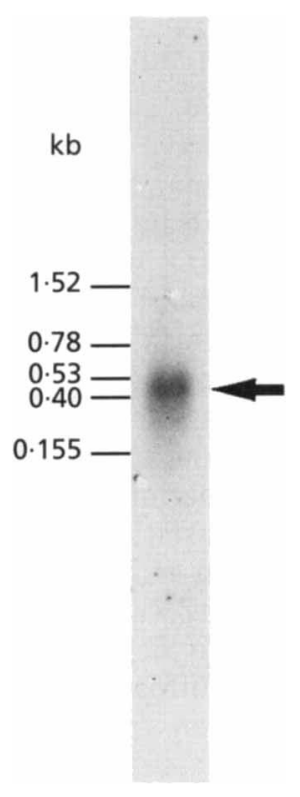

Fig. 2. Northern blot analysis of plasmodial RNA. Plasmodial RNA $(15 \mu \mathrm{g})$ was electrophoresed on a formaldehyde-agarose gel, transferred to a nylon membrane by capillary action and hybridized with the fluorescein-labelled CDNA insert.

ping regions and spanned a cDNA sequence of $408 \mathrm{bp}$ (Fig. 1). Hybridization of total RNA with a fluoresceinlabelled nucleotide oligomer from a cloned CDNA is shown in Fig. 2. The oligomer hybridized with a single RNA band of approximately $450 \mathrm{nt}$ (Fig. 2). The size of the RNA was consistent with the length of the cloned cDNA.

\section{cDNA sequence and deduced amino acid sequence}

The complete nucleotide sequence of the full-length cDNA contains $408 \mathrm{nt}$ with a protein-coding region of

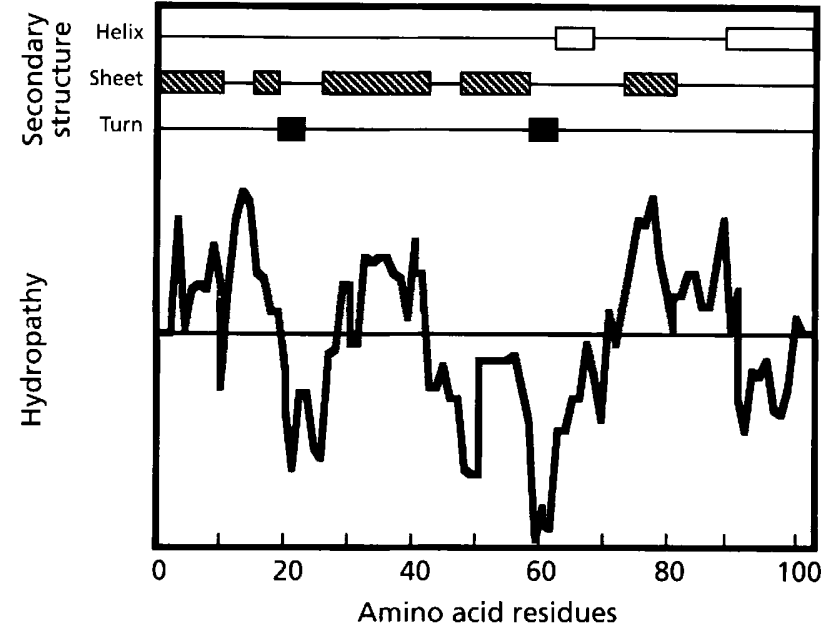

Fig. 3. Hydropathy profile (bottom) and prediction of $\alpha$-helices, $\beta$-sheets and $\beta$-turns (top) in the amino acid sequence of haemagglutinin I using the computer programs of Kyte \& Doolittle (1982) and Chou \& Fasman (1978), respectively. Data plotted above and below the vertical line indicate hydrophobic and hydrophilic portions, respectively. Predicted $\beta$-sheets and $\beta$ turns are shown by diagonal-lined and solid black boxes, respectively. Open boxes at the $C$ terminus indicate the predicted $\alpha$-helices.

$315 \mathrm{nt}$, beginning with an initiation codon, ATG, at positions 31-33 and ending with a stop codon, TAA, at positions $343-345$ (Fig. 1). The fragment has a $5^{\prime}$ noncoding region, as well as a $3^{\prime}$ non-coding region before the poly (A) tail containing a typical polyadenylation signal, AATAAA, at positions 366-371. The ORF specified a protein whose amino acid sequence matched that determined by Edman degradation (Table 2). We therefore identified this gene as that of haemagglutinin I. The $315 \mathrm{bp}$ ORF encodes a polypeptide of $104 \mathrm{aa}$ without a typical signal sequence. Lack of a secretory signal peptide in haemagglutinin I might be evidence that it is a cytosolic protein (Morita \& Nishi, 1992). The $\mathrm{N}$-terminal amino acid sequence from Edman degradation coincided with the deduced sequence starting from valine at the second residue, indicating the removal of the N-terminal methionine residue. ESI-MS analysis revealed that haemagglutinin $I$ has an apparent molecular mass of $10759 \cdot 86 \pm 0 \cdot 15 \mathrm{Da}$, corresponding exactly to that predicted from the cDNA sequence $(10760.76 \mathrm{Da})$. This suggests that haemagglutinin $\mathrm{I}$ is not post-translationally modified, except for the removal of the initiation methionine. Therefore, two consensus sequences (Asn-X-Ser/Thr) for the attachment of asparagine-linked oligosaccharide at amino acid residues 23-25 and 84-86 (Fig. 1) will be not glycosylated. The appearance of a phenylthiohydantoin-amino acid peak in the normal cycle by Edman degradation provides additional evidence for the lack of post-translational modification. The theoretical isoelectric point ( $\mathrm{pI}$ ) of the predicted amino acid sequence of haemagglutinin $\mathrm{I}$ is $6 \cdot 32$, consistent with the value of 6.9 determined by twodimensional IEF/SDS-PAGE (data not shown). Haema- 


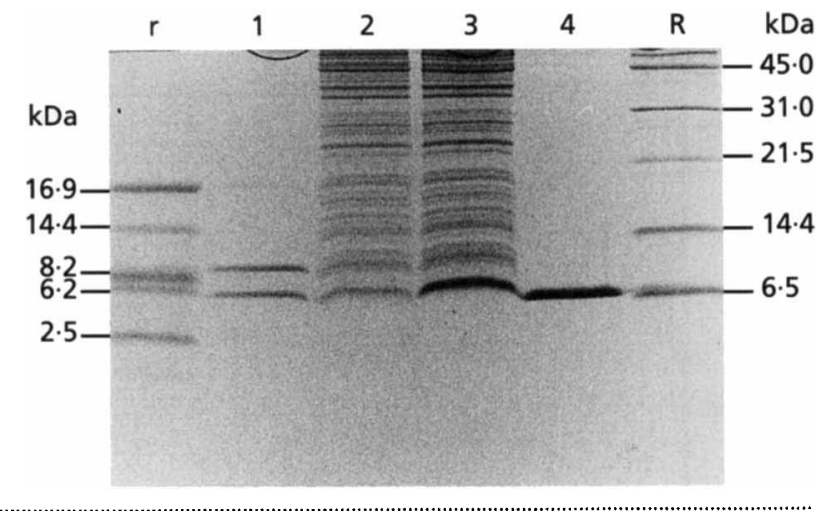

Fig. 4. Expression of recombinant haemagglutinin I in E. coli. Protein samples were analysed by Tricine-SDS-PAGE, followed by staining with Coomassie brilliant blue R-250. Lanes: 1, haemagglutinin I (lower) and II (upper) purified from plasmodia; $2, E$. coli cell lysate before induction; $3, E$. coli cell lysate after induction with $0.4 \mathrm{mM}$ IPTG; 4, purified recombinant haemagglutinin 1. Positions of molecular mass markers are indicated. Molecular mass markers (R) used were phosphorylase b $(97400 \mathrm{kDa})$, bovine serum albumin (66200), ovalbumin (45000), carbonic anhydrase (31000), soybean trypsin inhibitor (21500) and lysozyme (14400). Myoglobin fragments $(r)$ were also run on the same gel.

gglutinin $\mathbf{I}$ is rich in hydrophobic amino acid residues such as glycine $(13 \cdot 59 \%)$, alanine $(9 \cdot 71 \%)$ and valine $(9.71 \%)$ but no cysteine or methionine residues are found in the amino acid composition. A predominantly hydrophilic core of haemagglutinin I was observed at residues 42-70 (Fig. 3). The predicted secondary structure, analysed by the methods of Chou \& Fasman (1978), suggests that haemagglutinin I is mainly built up of $\beta$-sheets and two $\alpha$-helices (Fig. 3). Upon Tricine-SDSPAGE, haemagglutinin I gave a single band with a relative molecular mass of about $6 \mathrm{kDa}$, though ESI-MS analysis revealed a mass of $10759.86 \mathrm{Da}$. It is unlikely that the protein was digested by proteolytic enzymes during gel electrophoresis because the migration position was not changed in the presence of various proteinase inhibitors. The reason for the anomalously high electrophoretic mobility of haemagglutinin I is not clear at present.

The amino acid sequence of haemagglutinin I was subjected to comparative analysis with the entire SWISSPROT and NBRF sequence databases and the translated GenBank database for possible homologies between haemagglutinin I and other known proteins, including lectins. No significant sequence similarity was found between haemagglutinin I and other plant, animal and fungal lectins, and the bulk of other proteins examined.

\section{Expression of haemagglutinin I in E. coli}

Expression of haemagglutinin I in E. coli was performed using a bacteriophage T7 RNA polymerase/promoter system by induction with IPTG. Fig. 4 shows the results of Tricine-SDS-PAGE analysis of lysates of $E$. coli
BL21(DE3) containing pET with haemagglutinin I cDNA before (lane 2) and after (lane 3) induction with IPTG. E. coli appeared to produce a recombinant protein (lane 3), the position of which corresponds to that of native haemagglutinin I (lower band in lane 1). The recombinant protein, purified using a thyroglobulin-immobilized Toyopearl column, was detected as a single band (lane 4). The recombinant protein with marked haemagglutinating activity was isolated as a soluble protein at a yield of $3 \mathrm{mg}$ (litre culture) $)^{-1}$. The haemagglutinating activity of the recombinant protein was not inhibited by simple sugars (at $0.2 \mathrm{M}$ ) and glycoproteins such as ovalbumin, ovomucoid and mucin (at $5 \mathrm{mg} \mathrm{ml}^{-1}$ ). However, the activity was inhibited by thyroglobulin, orsomucoid and fetuin, the minimum amounts of which for inhibiting four haemagglutinating doses were $0.24,0.73$ and $1.61 \mathrm{mg} \mathrm{ml}^{-1}$, respectively. The inhibitory activity of these glycoproteins on recombinant haemagglutinin I is very similar to that on natural haemagglutinin I but not haemagglutinin II (Morita \& Nishi, 1992).

\section{DISCUSSION}

In this study we have cloned a cDNA encoding haemagglutinin I and have obtained over $0.4 \mathrm{kbp}$ of sequence information, spanning the protein-coding region and the $5^{\prime}$ and $3^{\prime}$ untranslated regions. Haemagglutinin I consists of 103 aa with a molecular mass of $10760.76 \mathrm{Da}$ and the primary structure indicates the lack of a typical signal sequence. Expression of cDNA in $E$. coli revealed that the recombinant protein has a molecular mass and a sugar-binding specificity similar to that of native haemagglutinin I. This result indicates that the cDNA clone obtained is truly that for haemagglutinin I and post-translational modifications such as $N$-glycosylation (Gowda et al., 1994), C-terminal proteolytic cleavage (Young et al., 1995) or N-terminal acetylation (Hirabayashi \& Kasai, 1993) are not essential for the haemagglutinating activity. Lack of posttranslational modification was demonstrated by comparing the molecular masses deduced from the cDNA with those determined by ESI-MS.

Computer analysis revealed that haemagglutinin I has not been classified into any lectin family such as animal C-type lectin, galectin and plant legume lectin. However, a comparison of the amino acid composition and the secondary structure of haemagglutinin I with those of other lectins revealed some similar features. These include the abundance of glycine, alanine, aspartic acid/asparagine, threonine and valine residues, the absence or low number of methionine, cysteine and arginine residues and the presence of an extensive network of $\beta$-sheets. The small number of sulphurcontaining amino acids has been reported in plant legume lectins (Sharon, 1993), fungal lectins (Rosen et al., 1992; Vranken et al., 1987; Yatohgo et al., 1988) and bacterial lectins (Avichezer et al., 1992). The lack of cysteine residues in haemagglutinin I supports the result that the haemagglutinating activity is not affected by 
thiol reagents (Morita \& Nishi, 1992). The major secondary structure in soluble plant lectins (Van Damme et al., 1996; Young et al., 1989) and animal galectins (Lobsanov et al., 1993; Muramoto \& Kamiya, 1992) is the $\beta$-sheet. Similarly, the presence of an extensive network of $\beta$-sheets bound by $\alpha$-helices has been shown in both Pseudomonas aeruginosa lectin (Avichezer et al., 1994) and Arthrobotrys oligospora lectin (Rosen et al., 1996b). These features were also found in the predicted secondary structure of haemagglutinin I (Fig. 3).

Many saline-soluble lectins with a relatively low molecular mass and low affinity to monosaccharides have been found in several fungal species. Mushroom lectins in Flammulina velutipes (Yatohgo et al., 1988) and Coprinus cinereus (Cooper et al., 1997) are developmentally regulated with high levels of expression in fruiting bodies and low levels of expression in vegetative mycelia, suggesting that these lectins may function in fruiting body formation. In P. polycephalum, however, it is unlikely that haemagglutinin I plays a role in the differentiation of plasmodia because acceleration of haemagglutinin synthesis was not always observed during macrocyst formation and fruiting body formation (Morita et al., 1995). Discoidin I, a lectin in the cellular slime mould $D$. discoideum, has an RGD sequence which participates in cell-substratum attachment (Barondes \& Springer, 1987). The RGD sequence, a cell-binding signal common to eukaryotic cell adhesion proteins, has also been found in several lectins (Ozeki et al., 1991; Relman et al., 1989). However, in spite of the developmental relationship to $D$. discoideum, no RGD sequence was found in Physarum haemagglutinin I. We therefore think that haemagglutinin I fulfils other important roles in this particular stage of the life cycle of this slime mould. As shown for galectin (Barondes et al., 1994), several fungal lectins (Cooper et al., 1997; Rosen et al., 1996b) have been reported to accumulate extracellularly despite the lack of a classical secretion signal sequence. The lack of a typical signal sequence in haemagglutinin I is notable because previous studies have indicated that haemagglutinin I is present in the slime layer that exists on the surface of the plasmodia (Morita \& Nishi, 1991). It is likely that haemagglutinin I is also externalized by non-classical mechanisms and functions in some physiological events by interaction with glycoconjugates on the plasmodial surface. Because of its preferential binding to acidic polysaccharide from the bacterial cell wall, we assume that the function of haemagglutinin may be to interact with other organisms (Morita et al., 1995). In spite of several similarities, the amino acid sequence of haemagglutinin $I$ is unrelated to those of fungal lectins and therefore represents a novel type of lectin.

In this study, we have expressed haemagglutinin I cDNA in E. coli BL21(DE3)plysS using the T7 RNA polymerase expression system. Site-directed mutagenesis using this system will provide us with valuable information concerning lectin-carbohydrate recognition. In addition, the isolation of haemagglutinin I cDNA will facilitate the investigation of several aspects of lectin function, including the regulation of its expression in growth and differentiation.

\section{ACKNOWLEDGEMENTS}

I thank Dr Shoji Okamura and Dr Teruhito Yasui for their helpful discussions and advice during the course of this investigation. I thank Naoko Shimada and Yukiko Iwado for their technical assistance.

\section{REFERENCES}

Avichezer, D., Katcoff, D. J., Garber, N. C. \& Gilboa-Garber, N. (1992). Analysis of the amino acid sequence of the Pseudomonas aeruginosa galactophilic PA-I lectin. J Biol Chem 267, 23023-23027.

Avichezer, D., Gilboa-Garber, N., Garber, N. C. \& Katcoff, D. J. (1994). Pseudomonas aeruginosa PA-I lectin gene molecular analysis and expression in Escherichia coli. Biochim Biophys Acta 1218, 11-20.

Barondes, S. H. \& Springer, W. R. (1987). An endogenous lectin and an oligosaccharide participate in adhesion mechanisms in Dictyostelium. In Genetic Regulation of Development, pp. 129-140. Edited by W. F. Loomis. New York: Alan R. Liss.

Barondes, S. H., Cooper, D. N. W., Gitt, M. A. \& Leffler, H. (1994). Galectins. Structure and function of a large family of animal lectins. J Biol Chem 269, 20807-20810.

Chou, P. Y. \& Fasman, G. D. (1978). Prediction of the secondary structure of proteins from their amino acid sequence. $A d v$ Enzymol 47, 45-148.

Cooper, D. N. W., Lee, S.-C. \& Barondes, S. H. (1983). Discoidinbinding polysaccharide from Dictyostelium discoideum. J Biol Chem 258, 8745-8750.

Cooper, D. N. W., Boulianne, R. P., Charlton, S., Farrell, E. M., Sucher, A. \& Lu, B. C. (1997). Fungal galectins, sequence and specificity of two isolectins from Coprinus cinereus. J Biol Chem 272, 1514-1521.

Daniel, J. W. \& Baldwin, H. H. (1964). Axenic culture of Physarum polycephalum on soluble media. Methods Cell Physiol 1, 15-29.

Frohman, M. A., Dush, M. K. \& Martin, G. R. (1988). Rapid production of full-length cDNAs from rare transcripts: amplification using a single gene-specific oligonucleotide primer. Proc Natl Acad Sci USA 85, 8998-9002.

Gowda, L. R., Savithri, H. S. \& Rao, D. R. (1994). The complete primary structure of a unique mannose/glucose-specific lectin from Field Bean (Dolichos Lab lab). J Biol Chem 269, 1878918793.

Hirabayashi, J. \& Kasai, K. (1993). The family of metazoan metalindependent beta-galactoside-binding lectins : structure, function and molecular evolution. Glycobiology 3, 297-304.

Inbar, J. \& Chet, I. (1994). A newly isolated lectin from the plant pathogenic fungus Sclerotium rolfsii: purification, characterization and role in mycoparasitism. Microbiology 140, 651-657.

Kyte, J. \& Doolittle, R. F. (1982). A simple method for displaying the hydropathic character of a protein. J Mol Biol 157, 105-132.

Lobsanov, Y. D., Gitt, M. A., Leffler, H., Barondes, S. H. \& Rini, J. M. (1993). X-ray crystal structure of the human dimeric S-Lac lectin, L-14-II, in complex with lactose at $2 \cdot 9$ - $\AA$ resolution. $J$ Biol Chem 268, 27034-27038.

Matsudaira, P. (1987). Sequence from picomole quantities of proteins electroblotted onto polyvinylidene difluoride membranes. J Biol Chem 262, 10035-10038. 
Morita, M. \& Nishi, A. (1991). Glycoproteins and enzymes associated with plasmodial membrane and slime layer of $P h y$ sarum polycephalum. J Gen Appl Microbiol 37, 93-109.

Morita, M. \& Nishi, A. (1992). Isolation and partial characterization of haemagglutinins from plasmodia of Physarum polycephalum. J Gen Microbiol 138, 619-625.

Morita, M., Minami, E., Zheng, L. \& Nishi, A. (1995). Physarum polycephalum haemagglutinins: effect of nutrition on synthesis, and their possible role in nature. Microbiology 141, 2315-2322.

Muramoto, K. \& Kamiya, H. (1992). The amino-acid sequence of a lectin from conger eel, Conger myriaster, skin mucus. Biochim Biophys Acta 1116, 129-136.

Nishimura, M., Tanigaki, C. \& Okamura, S. (1991). Changes in $\beta$ tubulin isoforms and their RNA level in synchronized tobacco cells. Cell Struct Funct 16, 489-494.

Ozeki, Y., Matsui, T., Suzuki, M. \& Titani, K. (1991). Amino acid sequence and molecular characterization of a D-galactosidespecific lectin purified from sea urchin (Anthocidaris crassispina) eggs. Biochemistry 30, 2391-2394.

Relman, D. A., Domenighini, M., Tomanen, E., Rappuoli, R. \& Falkow, S. (1989). Filamentous hemagglutinin of Bordetella pertussis: nucleotide sequence and crucial role in adherence. Proc Natl Acad Sci USA 86, 2637-2641.

Rosen, S., Ek, L., Rask, L. \& Tunlid, A. (1992). Purification and characterization of a surface lectin from the nematode-trapping fungus Arthrobotrys oligospora. J Gen Microbiol 138, 2663-2672.

Rosen, S., Bergstrom, J., Karlsson, K.-A. \& Tunlid, A. (1996a). A multispecific saline-soluble lectin from the parasitic fungus Arthrobotrys oligospora. Similarities in the binding specificities compared with a lectin from the mushroom Agaricus bisporus. Eur J Biochem 238, 830-837.

Rosen, S., Kata, M., Persson, Y., Lipniunas, P. H., Wikstrom, M., van den Hondel, C. A. M. J. J., van den Brink, J. M., Rask, L., Heden, L.-O. \& Tunlid, A. (1996b). Molecular characterization of a saline-soluble lectin from a parasitic fungus. Extensive sequence similarities between fungal lectins. Eur J Biochem 238, 822-829.

Sambrook, J., Fritsch, E. F. \& Maniatis, T. (1989). Molecular Cloning: a Laboratory Manual, 2nd edn. Cold Spring Harbor, NY: Cold Spring Harbor Laboratory.
Sanger, F., Nicklen, S. \& Coulson, A. R. (1977). DNA sequencing with chain-terminating inhibitors. Proc Natl Acad Sci USA 74, 5463-5467.

Schagger, H. \& van Jagow, G. (1987). Tricine-sodium dodecyl sulfate-polyacrylamide gel electrophoresis for the separation of proteins in the range from 1 to $100 \mathrm{kDa}$. Anal Biochem 166 , 368-379.

Schreckenbach, T. \& Werenskiold, A.-K. (1986). Gene expression during plasmodial differentiation. In The Molecular Biology of Physarum polycephalum, pp. 131-150. Edited by W. F. Dove, J. Dee, S. Hatano, F. B. Haugli \& K.-E. Wohlfarth-Bottermann. New York: Plenum Press.

Sharon, N. (1993). Lectin-carbohydrate complexes of plants and animals: an atomic view. Trends Biochem Sci 18, 221-226.

Van Damme, E. J. M., Barre, A., Verhaert, P., Rouge, P. \& Peumans, W. J. (1996). Molecular cloning of the mitogenic mannose/maltose-specific rhizome lectin from Calystegia sepium. FEBS Lett 397, 352-356.

Vranken, A. M., Van Damme, E. J. M., Allen, A. K. \& Peumans, W. J. (1987). Purification and properties of an $\mathrm{N}$-acetylgalactosamine specific lectin from the plant pathogenic fungus Rbizoctonia solani. FEBS Lett 216, 67-72.

Yatohgo, T., Nakata, M., Tumuraya, Y., Hashimoto, Y. \& Yamamoto, S. (1988). Purification and properties of a lectin from the fruitbodies of Flammulina velutipes. Agric Biol Chem 52, 1485-1493.

Young, N. M., Johnston, R. A. Z., Szabo, A. G. \& Watson, D. C. (1989). Homology of the D-galactose-specific lectins from Artocarpus integrifolia and Maclura pomifera and the role of an unusual small polypeptide subunit. Arch Biochem Biophys 270, 596-603.

Young, N. M., Watson, D. C., Yaguchi, M., Ader, R., Arango, R., Rodriguez-Arango, E., Sharon, N., Blay, P. K. S. \& Thibault, P. (1995). C-Terminal post-translational proteolysis of plant lectins and their recombinant forms expressed in Escherichia coli. J Biol Chem 270, 2563-2570.

Received 5 September 1997; revised 6 November 1997; accepted 24 December 1997. 